\title{
OPTIMAL CONTROL PROBLEMS FOR GENERAL GLOBAL HYBRID DYNAMICAL SYSTEMS WITH MATRIX COST FUNCTIONAL
}

\author{
RUI GAO ${ }^{\otimes 1}$ and XINZHI LIU ${ }^{2}$ \\ (Received 22 April, 2009; revised 29 January, 2010)
}

\begin{abstract}
This paper considers an optimal control problem for a class of controlled hybrid dynamical systems (HDSs) with prescribed switchings. By using Ekeland's variational principle and a matrix cost functional, a minimum principle for HDSs is derived, which provides a necessary condition of the aforementioned problem. The results given in this paper include both pure continuous systems and pure discrete-time systems as special cases.
\end{abstract}

2000 Mathematics subject classification: primary 49J21; secondary 34A38.

Keywords and phrases: hybrid dynamical system, optimal control, minimum principle, Ekeland's variational principle, restricted terminal states.

\section{Introduction}

A hybrid dynamical system (HDS) contains continuous variable dynamical systems (CVDSs) and discrete event dynamical systems (DEDSs) that interact with each other. There are several classes of HDS. A typical class is the switching system, which is capable of exhibiting simultaneously several kinds of dynamic behaviour in different parts of the system. Optimal control problems involving HDSs have been studied broadly in recent years. Branicky et al. $[5,7]$ proposed a unified framework of hybrid optimal control and synthesized a hybrid controller for hybrid devices. Then they demonstrated the existence of optimal (relaxed) and near-optimal (piecewise) controls and derived "generalized quasi-variational inequalities" that can be solved by algorithms based on a generalized Bellman equation, impulse control and linear programming. Liu et al. [18] developed an algorithm for a class of nonlinear impulsive HDS. Furthermore, this algorithm was recently extended by Loxton et al. [19, 20] to

\footnotetext{
${ }^{1}$ School of Control Science \& Engineering, Shandong University, 250061 Ji'nan, PR China; e-mail: gaorui@sdu.edu.cn.

${ }^{2}$ Department of Applied Mathematics, University of Waterloo, Waterloo, Ontario, Canada N2L 3G1; e-mail: xzliu@waterloo.ca.

(C) Australian Mathematical Society 2010, Serial-fee code 1446-1811/2010 \$16.00
} 
solve impulsive switched system optimizations. Pepyne and Cassandras [8, 24, 25] constructed optimal control frameworks of HDSs for a manufacturing process model. Schutter [26] considered a class of queueing systems and presented methods to determine the optimal switching instants to minimize a criterion such as average queue length, worst-case queue length, average waiting time, and so on. Then it was shown that, if there was no upper saturation, for some objective functions the optimal switching scheme could be computed. Hedlund and Rantzer [15] proposed a method for optimal control of HDSs based on an inequality of Bellman and convex optimization, then gave a lower bound for the optimal value function. Bemporad and Morari [2] transformed a class of HDSs into a mixed logical dynamical system and then dealt with its optimization problem by mixed integer quadratic programming.

Guia et al. [12] studied the optimal control problem of minimizing a quadratic performance index over an infinite time horizon for a class of switched piecewise linear autonomous systems. Tan et al. [31] concentrated on sampled data based on linear quadratic adaptive control of continuous-time systems with unknown Markov jump parameters, and then gave a parameter estimator and a control design method. Bengea and DeCarlo [3] considered an optimal control problem for a class of switching systems under the assumption that the number of switches and model sequences are both indeterminate. Baotic et al. [1] studied the constrained finite- and infinitetime optimal control problem for the class of discrete-time linear HDS and proposed algorithms that compute the optimal solution. Borrelli et al. [4] worked on the solution to optimal control problems for constrained discrete-time linear HDSs based on quadratic or linear performance criteria and constructed the state-feedback optimal control law by combining multi-parametric programming and dynamic programming. Gokbayrak and Selvi [13] derived some sample path characteristics for a two-stage serial HDS, and transformed an original nonsmooth optimal control problem into a convex optimization problem. Spinelli et al. [28] dealt with the problem of optimal control of continuous-time autonomous linear switched systems on a finite control horizon and developed sufficient conditions for their optimality using HamiltonJacobi-Bellman theory. Shaikh and Caines [27] studied a class of hybrid optimal control problems for systems with controlled and autonomous location transitions and extended the maximum principle from pure continuous systems to HDSs. Shaikh presented a set of necessary conditions of hybrid system trajectory optimality and a class of general hybrid maximum principle based algorithms.

Gao et al. [10, 11] studied optimal control problems concerning a class of HDSs with a pre-specified sequence of switched subsystems over a local interval for both free terminal states and restricted terminal states. Other problems related to HDSs have also been widely studied. Guan et al. [14] studied a class of hybrid impulsive and switching systems, and applied these systems to nonlinear control. Trecate et al. [32] presented methods for the analysis of discrete-time piecewise affine and hybrid systems. Stability of HDSs is an important research area. Branicky [6] proposed Lyapunov function analysis tools for switched and hybrid systems. Michel et al. [23, 30, 35] discussed stability theory for general HDSs. Xu and Zhai [33] considered practical stability 
and stabilization of hybrid and switched systems. Chai and Teel [9] proved that the existence of smooth Lyapunov functions for HDSs is equivalent to their robustness. Liu and Shen [17] studied the stability theory of HDSs with time delay. For the controllability of switching linear HDSs, Yang [34] proposed an algebraic approach, and Stikkel et al. [29] studied necessary and sufficient conditions for its applicability. Lazar et al. [16] studied stabilizing model predictive control for HDSs. Margaliot [21] did stability analysis of switched systems by variational principles. Meng and Zhang [22] studied output feedback based admissible control of the switched linear singular system.

In this paper, we continue the work of $[10,11]$. We impose an outer restriction upon the terminal states of the HDS and extend the aforementioned results from a certain continuous time interval to the whole time horizon. Also, we present the minimum principle of global HDSs and prove it by means of Ekeland's variational principle. The rest of the paper is organized as follows. In Section 2 we describe a general controlled model of HDSs and formulate the optimal control problem. Then we propose, in Section 3, a mathematical tool, that is, the minimum principle of HDSs, which can be used to establish the necessary conditions for the optimal control problems mentioned above. In the proof, Ekeland's variational principle and matrix cost functional expression are applied. In Section 4 we consider, as applications of our main results, some special cases and obtain some corresponding results for switched linear time-variant systems and pure discrete-time systems. The conclusion of the paper is set out in Section 5.

\section{Preliminaries}

2.1. Notation The set $R^{+}$represents the nonnegative real numbers, $Z^{+}$denotes the set of all nonnegative integers, and meas $(\Psi)$ denotes the measure of the set $\Psi$. Also useful is the differential operator $\nabla=(\partial / \partial x, \partial / \partial q)$.

2.2. Description of optimal control problems in global HDSs The controlled model of an HDS is given by

$$
\begin{cases}\dot{x}(t)=f\left(t, x(t), q\left(\tau_{k}\right), u(t)\right), & t \in\left[\tau_{k}, \tau_{k+1}\right), k \in Z^{+}, \\ q\left(\tau_{k+1}\right)=v\left(x\left(t^{-}\right), q\left(\tau_{k}\right), u\left(t^{-}\right)\right), & t=\tau_{k+1},\end{cases}
$$

where $x(t) \in \mathbb{R}^{n}$ denotes the state of the CVDS at $t$, and $q\left(\tau_{k}\right) \in \mathbb{R}^{s}$ denotes the state of the DEDS at $\tau_{k}$. $\tau_{k}$ denotes the $k$ th given switching instant, at which the state of the DEDS $q(\cdot)$ changes. We consider the HDS (2.1) evolving over $\left[\tau_{0}, t^{f}\right]$. We suppose that $\tau_{l}=\max \left\{\tau_{k} \in R^{+} \mid \tau_{k} \leq t^{f}, k \in Z^{+}\right\}$, and define the finite set of switching instants

$$
E \triangleq\left\{\tau_{k} \in R^{+} \mid k=0,1, \ldots, l ; 0 \leq \tau_{0}<\tau_{1}<\cdots<\tau_{l} \leq t^{f}\right\} .
$$

Moreover, $\left(t, \tau_{k}\right)$ denotes a time element of the HDS, which satisfies $t \in\left[\tau_{k}, \tau_{k+1}\right)$. In particular, we denote the switching instant by $\left(\tau_{k}, \tau_{k}\right)$. Corresponding to any $\left(t, \tau_{k}\right)^{T}$, 
the state of the HDS is denoted by $\left(x(t), q\left(\tau_{k}\right)\right)$. We define the control range as

$$
U \triangleq\left\{\left(u_{1}, u_{2}, \ldots, u_{m}\right)^{T} \in \mathbb{R}^{m}:\left|u_{i}\right| \leq 1, i=1,2, \ldots, m\right\} .
$$

The control input of the system is $u(t) \in U \subset \mathbb{R}^{m}$ for all $t \in R$. The mapping $f: R^{+} \times \mathbb{R}^{n} \times \mathbb{R}^{s} \times U \rightarrow \mathbb{R}^{n}$ is integrable with respect to $t$ and satisfies Lipschitz conditions with respect to $u$. Furthermore, $f$ has bounded partial derivatives with respect to $x$ and $q$. The mapping $v: \mathbb{R}^{n} \times \mathbb{R}^{s} \times U \rightarrow \mathbb{R}^{s}$ is continuous with respect to $u$, and has bounded partial derivatives with respect to $x$ and $q$.

Without loss of generality, we define the initial instant $(0,0)$ and the terminal instant $\left(t^{f}, \tau_{l}\right)$. Then, the terminal state of the HDS is $\left(x\left(t^{f}\right), q\left(\tau_{l}\right)\right)$. We assume that the HDS (2.1) runs from the initial state $(x(0), q(0))$, which ensures the existence and uniqueness of the solution of the system equation. Moreover, the time horizon can be divided into $l+1$ continuous subintervals, that is,

$$
\left[\tau_{0}, t^{f}\right]=\bigcup_{k=0}^{l-1}\left[\tau_{k}, \tau_{k+1}\right) \cup\left[\tau_{l}, t^{f}\right] .
$$

DEFINITION 1 (Admissible control). Let HDS (2.1) evolve over $\left[\tau_{0}, t^{f}\right]$ to terminal state $\left(x\left(t^{f}\right), q\left(\tau_{l}\right)\right)$. The control $u(\cdot)$ is called an admissible control if $u(\cdot):\left[\tau_{0}, t^{f}\right] \rightarrow$ $U$ is bounded and quadratically integrable and $G\left(x\left(t^{f}\right), q\left(\tau_{l}\right)\right)=0$, where $G(\cdot, \cdot)$ : $\mathbb{R}^{n} \times \mathbb{R}^{s} \rightarrow \mathbb{R}^{n_{1}}\left(n_{1} \leq n+s\right)$ is continuously differentiable in its arguments. The set of admissible controls is denoted by

$$
U_{a d} \triangleq\left\{u(\cdot) \in L\left[\tau_{0}, t^{f} ; U\right] \mid G\left(x\left(t^{f}\right), q\left(\tau_{l}\right)\right)=0 \text { under } u(\cdot)\right\} .
$$

Suppose that the HDS evolves under the controls $u_{k}(\cdot) \in U_{\mathrm{ad}}(k=0,1, \ldots, l)$. The trajectories of its CVDS and the discrete events are denoted by $x\left(\cdot, u_{k}(\cdot)\right)$ and $q\left(\cdot, u_{k}(\cdot)\right)$, respectively. We define the control input by

$$
\mathbf{u}(\cdot)=\left(u_{0}(\cdot), u_{1}(\cdot), \ldots, u_{l}(\cdot)\right)^{T} \in U_{\mathrm{ad}}^{l+1},
$$

and defining, for $\alpha=0, \ldots, l-1$,

$$
\begin{aligned}
\mathcal{T}_{\alpha}=\sum_{k=\alpha}^{l-1} & {\left[\int_{\tau_{k}}^{\tau_{k+1}} L\left(s, x\left(s, u_{\alpha}(s)\right), q\left(\tau_{k}, u_{\alpha}(s)\right), u_{\alpha}(s)\right) d s+L_{a}\left(q\left(\tau_{k+1}, u_{\alpha}(\cdot)\right)\right)\right] } \\
& +\int_{\tau_{l}}^{t^{f}} L\left(s, x\left(s, u_{\alpha}(s)\right), q\left(\tau_{l}, u_{\alpha}(s)\right), u_{\alpha}(s)\right) d s \\
& +g\left(x\left(t^{f}, u_{\alpha}(\cdot)\right), q\left(\tau_{l}, u_{\alpha}(\cdot)\right)\right)
\end{aligned}
$$

and

$$
\mathcal{T}_{l}=\int_{\tau_{l}}^{t^{f}} L\left(s, x\left(s, u_{l}(s)\right), q\left(\tau_{l}, u_{l}(s)\right), u_{l}(s)\right) d s+g\left(x\left(t^{f}, u_{l}(\cdot)\right), q\left(\tau_{l}, u_{l}(\cdot)\right)\right)
$$


allows the definition of the cost functional

$$
J(\boldsymbol{u}(\cdot))=\operatorname{diag}\left\{\mathcal{T}_{0}, \mathcal{T}_{1}, \ldots, \mathcal{T}_{l-1}, \mathcal{T}_{l}\right\},
$$

where $\operatorname{diag}(\cdot)$ denotes a diagonal matrix and $L: R^{+} \times \mathbb{R}^{n} \times \mathbb{R}^{s} \times \mathbb{R}^{m} \rightarrow R^{+}$ describes the running expenses of the HDS. For arbitrary $\left[\tau_{k}, \tau_{k+1}\right)$ for $k=$ $0,1, \ldots, l-1$ or $\left[\tau_{l}, t^{f}\right]$, the function $L$ is integrable with respect to $t$ and is continuous with respect to $u$. $L$ is continuously differentiable with respect to $x$ and $q$. The function $L_{a}: \mathbb{R}^{s} \rightarrow R^{+}$describes the switching cost of a DEDS, and $L_{a}$ is continuously differentiable in all its variables. Considering the terminal state of the HDS, we define a mapping function $g(\cdot, \cdot): \mathbb{R}^{n} \times \mathbb{R}^{s} \rightarrow R^{+}$, which is smooth in its arguments.

Then the optimal control problem of the HDS can be described as follows. We assume that there exists the optimal control $\bar{u}(\cdot) \in U_{\mathrm{ad}}$, and we define $\overline{\boldsymbol{u}}(\cdot)=$ $(\bar{u}(\cdot), \bar{u}(\cdot), \ldots, \bar{u}(\cdot))^{T}$, a vector of $l+1$ identical components, so that under the control of $\overline{\boldsymbol{u}}(\cdot)$ the cost functional (2.4) reaches its "minimum". That is, for any control vector $\boldsymbol{u}(\cdot) \in U_{\mathrm{ad}}^{l+1}$, the condition that $J(\boldsymbol{u}(\cdot))-J(\overline{\boldsymbol{u}}(\cdot))$ is positive semi-definite is satisfied.

In the following section, we will derive a necessary condition for the above global HDS optimal control problem-the minimum principle of HDS over the interval $\left[\tau_{0}, t^{f}\right]$.

\section{The minimum principle for HDSs with restricted terminal states}

DEFINITION 2 (Lebesgue point [36]). Given a Lebesgue integrable function $f$, a point $t$ in the domain of $f$ is a Lebesgue point if

$$
\lim _{r \rightarrow 0^{+}} \frac{1}{|B(t, r)|} \int_{B(t, r)}|f(s)-f(t)| d s=0,
$$

where $B(t, r)$ is the ball centred at $t$ with radius $r$ and Lebesgue measure $|B(t, r)|$.

LEMMA 3.1 (Ekeland's variational principle). Let $V$ be a complete metric space and $F: V \rightarrow R$ a lower semi-continuous function not identically equal to $+\infty$. Assume that $F$ is bounded below and that $\bar{u} \in V$ is an $\varepsilon$-minimum of $F$. That is, for a given $\varepsilon>0$ it satisfies

$$
F(\bar{u}) \leq \inf _{\nu \in V} F(v)+\varepsilon .
$$

Then there exists $\tilde{u} \in V$ and $\tilde{u} \neq \bar{u}$ such that $F(\tilde{u}) \leq F(\bar{u})$ and $d(\bar{u}, \tilde{u}) \leq \varepsilon$. For any $\nu \in V, F(\nu) \geq F(\tilde{u})-\sqrt{\varepsilon} d(\nu, \tilde{u})$.

LEMMA 3.2. Let $f:[a, b] \rightarrow \mathbb{R}^{n}$ be a Lebesgue integrable function, $\lambda \in(0,1)$, for any $\varepsilon>0$; then there exists a measurable set $E_{\lambda}(\varepsilon) \subset[a, b]$, such that

$$
\operatorname{meas}\left(E_{\lambda}(\varepsilon)\right)=\lambda(b-a), \quad \lambda \int_{a}^{b} f(t) d t=\int_{E_{\lambda}(\varepsilon)} f(t) d t+\eta, \quad\|\eta\|<\varepsilon .
$$

For more details about Lemmas 3.1 and 3.2, the interested reader is referred to [36]. 
THEOREM 3.3 (Minimum principle of global HDS). Let $\bar{u}(\cdot)$ be a solution of the HDS (2.1). We suppose that $x(\cdot)=x(\cdot, \bar{u}(\cdot))$ and $q(\cdot)=q(\cdot, \bar{u}(\cdot))$ are the optimal trajectories of the continuous subsystems and optimal discrete events' states, respectively, corresponding to the optimal control $\bar{u}(\cdot)$. Then there exist $h_{k}$ and piecewise continuous $y_{k}(\cdot)$ that satisfy the adjoint equations

$$
-\frac{d}{d t} y_{k}(t)=\frac{\partial^{T}}{\partial x} f\left(t, x(t), q\left(\tau_{k}\right), \bar{u}(t)\right) y_{k}(t)+h_{k} \frac{\partial}{\partial x} L\left(t, x(t), q\left(\tau_{k}\right), \bar{u}(t)\right),
$$

over $\left[\tau_{k}, \tau_{k+1}\right)$ for $k=0,1, \ldots, l-1$, or over $\left[\tau_{l}, t^{f}\right]$ for $k=l$. There exists an $n_{1}$-dimensional vector $\psi_{k}$, which satisfies

$$
h_{k}^{2}+\left\|\psi_{k}\right\|^{2}=1
$$

such that at $t^{f}, y_{k}(\cdot)$ satisfies

$$
y_{k}\left(t^{f}\right)=\frac{\partial^{T}}{\partial x} G\left(x\left(t^{f}\right), q\left(\tau_{l}\right)\right) \psi_{k}+h_{k} \frac{\partial}{\partial x} g\left(x\left(t^{f}\right), q\left(\tau_{l}\right)\right) .
$$

Over the same interval, the minimum condition

$$
\begin{aligned}
& h_{k} L\left(t, x(t), q\left(\tau_{k}\right), \bar{u}(t)\right)+\left\langle y_{k}(t), f\left(t, x(t), q\left(\tau_{k}\right), \bar{u}(t)\right)\right\rangle \\
& \quad=\min _{u \in U}\left\{h_{k} L\left(t, x(t), q\left(\tau_{k}\right), u\right)+\left\langle y_{k}(t), f\left(t, x(t), q\left(\tau_{k}\right), u\right)\right\rangle\right\}
\end{aligned}
$$

holds almost everywhere.

PROOF. We define Ekeland's distance in $U_{\text {ad }}$ : for any $u_{1}(\cdot), u_{2}(\cdot) \in U_{\text {ad }}$,

$$
d\left(u_{1}(\cdot), u_{2}(\cdot)\right)=\operatorname{meas}\left\{t \in\left[0, t^{f}\right]: u_{1}(t) \neq u_{2}(t)\right\},
$$

and it has been proven that $U_{\text {ad }}$ is complete under this measure when the control set $U$ is defined by (2.2) [36].

Let $\rho>0$; for a given $\boldsymbol{u}(\cdot) \in U_{\text {ad }}^{l+1}$ we define a new cost functional

$$
\begin{aligned}
J_{\rho}^{2}(\boldsymbol{u}(\cdot)) & \\
\quad= & \operatorname{diag}\left\{\left\|G\left(x\left(t^{f}, u_{0}(\cdot)\right), q\left(\tau_{l}, u_{0}(\cdot)\right)\right)\right\|^{2}, \ldots,\left\|G\left(x\left(t^{f}, u_{l}(\cdot)\right), q\left(\tau_{l}, u_{l}(\cdot)\right)\right)\right\|^{2}\right\} \\
& +[J(\boldsymbol{u}(\cdot))-J(\overline{\boldsymbol{u}}(\cdot))+E \sqrt{\rho}]^{2},
\end{aligned}
$$

where $E$ is the $(l+1) \times(l+1)$ identity matrix. We have that $J_{\rho}(\boldsymbol{u}(\cdot)): U_{\mathrm{ad}}^{l+1} \rightarrow \mathbb{R}^{l+1}$ is a continuous matrix functional, and $J_{\rho}^{2}(\boldsymbol{u}(\cdot)) \geq 0$ for all $\boldsymbol{u}(\cdot) \in U_{\mathrm{ad}}^{l+1}$. Then

$$
J_{\rho}^{2}(\overline{\boldsymbol{u}}(\cdot)) \leq \inf _{\boldsymbol{u}(\cdot) \in U_{\mathrm{ad}}}\left\{J_{\rho}^{2}(\boldsymbol{u}(\cdot))\right\}+E \rho .
$$

By Lemma 3.1, there must be $\boldsymbol{u}_{\rho}(\cdot) \neq \overline{\boldsymbol{u}}(\cdot)$ such that $J_{\rho}(\overline{\boldsymbol{u}}(\cdot))-J_{\rho}\left(\boldsymbol{u}_{\rho}(\cdot)\right)$ is positive definite and

$$
d\left(u_{\rho i}(\cdot), \bar{u}(\cdot)\right) \leq \rho, \quad i=0,1, \ldots, l .
$$


For any $\boldsymbol{u}(\cdot)$, we obtain that

$$
J_{\rho}(\boldsymbol{u}(\cdot))-J_{\rho}\left(\boldsymbol{u}_{\rho}(\cdot)\right)+E \sqrt{\rho} d\left(\boldsymbol{u}_{\rho}(\cdot), \boldsymbol{u}(\cdot)\right)
$$

is positive semi-definite. Now for sets $E_{k \varepsilon}(k=0,1, \ldots, l-1)$ measurable on $\left[\tau_{k}, \tau_{k+1}\right)$ and $E_{l \varepsilon}$ measurable on $\left[\tau_{l}, t^{f}\right]$ such that

$$
\begin{aligned}
& \operatorname{meas}\left(E_{k \varepsilon}\right)=\varepsilon\left(\tau_{k+1}-\tau_{k}\right), \quad k=0,1, \ldots, l-1, \\
& \operatorname{meas}\left(E_{l \varepsilon}\right)=\varepsilon\left(t^{f}-\tau_{l}\right),
\end{aligned}
$$

construct a new set of controls $\boldsymbol{u}_{\rho}^{\varepsilon}(\cdot)=\left(u_{\rho 0}^{\varepsilon}(\cdot), u_{\rho 1}^{\varepsilon}(\cdot), \ldots, u_{\rho l}^{\varepsilon}(\cdot)\right)^{T}$ by

$$
u_{\rho k}^{\varepsilon}(t)=\left\{\begin{array}{ll}
u_{k}^{0}(t), & t \in E_{k \varepsilon} \subset\left[\tau_{k}, \tau_{k+1}\right), \\
u_{\rho k}^{0}(t), & t \in\left[\tau_{0}, t^{f}\right] \backslash E_{k \varepsilon},
\end{array} \quad k=0,1, \ldots, l-1,\right.
$$

and

$$
u_{\rho l}^{\varepsilon}(t)= \begin{cases}u_{l}^{0}(t), & t \in E_{l \varepsilon} \subset\left[\tau_{l}, t^{f}\right] \\ u_{\rho l}^{0}(t), & t \in\left[\tau_{0}, t^{f}\right] \backslash E_{l \varepsilon}\end{cases}
$$

If $u_{k}(\cdot) \in U_{\text {ad }}$ and $\varepsilon$ is small enough, we know that $u_{k}(\cdot)$ is square integrable in $E_{k \varepsilon}$. That is, $u_{\rho k}^{\varepsilon}(\cdot) \in U_{\mathrm{ad}}$. By (3.5),

$$
J_{\rho}\left(\boldsymbol{u}_{\rho}^{\varepsilon}(\cdot)\right)-J_{\rho}\left(\boldsymbol{u}_{\rho}(\cdot)\right)+E \sqrt{\rho} d\left(\boldsymbol{u}_{\rho}(\cdot), \boldsymbol{u}(\cdot)\right)
$$

is positive definite. For simplicity, we discuss only the $k$ th diagonal element.

We suppose that the continuous states of the subsystems are $x_{\rho k}^{\varepsilon}(\cdot), x_{\rho k}^{0}(\cdot)$, and the discrete events' states are $q_{\rho k}^{\varepsilon}(\cdot), q_{\rho k}^{0}(\cdot)$, corresponding to the controls $u_{\rho k}^{\varepsilon}(\cdot)$ and $u_{\rho k}^{0}(\cdot)$, respectively. For $t \in\left[\tau_{k}, \tau_{k+1}\right), x_{\rho k}^{\varepsilon}(\cdot)$ and $x_{\rho k}^{0}(\cdot)$ both start at the initial state $x_{\rho k}^{0}\left(\tau_{k}\right)$ (because of the continuity of the CVDS trajectories and $u_{\rho k}^{\varepsilon}(t)=u_{\rho k}^{0}(t)$ for $\left.t \in\left[0, \tau_{k}\right)\right)$, and defining

$$
f^{\phi, \xi}\left(\cdot, \tau_{k}\right)=f\left(\cdot, x_{\rho k}^{\phi}(\cdot), q_{\rho k}^{0}\left(\tau_{k}\right), u_{\rho k}^{\xi}(\cdot)\right), \quad \phi, \xi=0, \varepsilon,
$$

they satisfy

$$
x_{\rho k}^{\phi}(t)=x_{\rho k}^{0}\left(\tau_{k}\right)+\int_{\tau_{k}}^{t} f^{\phi, \phi}\left(s, \tau_{k}\right) d s, \quad \phi=0, \varepsilon, t \in\left[\tau_{k}, \tau_{k+1}\right) .
$$

Let

$$
\begin{gathered}
\Delta x_{k}(\cdot)=x_{\rho k}^{\varepsilon}(\cdot)-x_{\rho k}^{0}(\cdot), \quad z_{k}^{\varepsilon}(\cdot)=\frac{1}{\varepsilon} \Delta x_{k}(\cdot), \\
\Delta f_{k}(\cdot)=f\left(\cdot, x_{\rho k}^{0}(\cdot), q_{\rho k}^{0}\left(\tau_{k}\right), u_{k}^{0}(\cdot)\right)-f^{0,0}\left(\cdot, \tau_{k}\right)
\end{gathered}
$$

and

$$
\hat{f}_{k}(\cdot)=f\left(\cdot, x_{\rho k}^{0}(\cdot)+\lambda\left(x_{\rho k}^{\varepsilon}(\cdot)-x_{\rho k}^{0}(\cdot)\right), q_{\rho k}^{0}\left(\tau_{k}\right), u_{\rho k}^{\varepsilon}(\cdot)\right)
$$


By Lemma 3.2, we get

$$
\begin{aligned}
z_{k}^{\varepsilon}(t) & =\frac{1}{\varepsilon} \int_{\tau_{k}}^{t}\left[f^{\varepsilon, \varepsilon}\left(s, \tau_{k}\right)-f^{0,0}\left(s, \tau_{k}\right)\right] d s \\
& =\frac{1}{\varepsilon} \int_{\tau_{k}}^{t}\left[f^{\varepsilon, \varepsilon}\left(s, \tau_{k}\right)-f^{0, \varepsilon}\left(s, \tau_{k}\right)\right] d s+\frac{1}{\varepsilon} \int_{\tau_{k}}^{t}\left[f^{0, \varepsilon}\left(s, \tau_{k}\right)-f^{0,0}\left(s, \tau_{k}\right)\right] d s \\
& =\int_{\tau_{k}}^{t} \int_{0}^{1} \frac{\partial \hat{f}_{k}(s)}{\partial x} \frac{1}{\varepsilon} \Delta x_{k}(s) d \lambda d s+\frac{1}{\varepsilon} \int_{E_{k \varepsilon} \bigcap\left[\tau_{k}, t\right)} \Delta f_{k}(s) d s \\
& =\int_{\tau_{k}}^{t}\left[\int_{0}^{1} \frac{\partial \hat{f}_{k}(s)}{\partial x} d \lambda\right] z_{k}^{\varepsilon}(s) d s+\int_{\tau_{k}}^{t} \Delta f_{k}(s) d s+o(\varepsilon)
\end{aligned}
$$

Letting $\varepsilon \rightarrow 0$, the integral of the first term in (3.9) has a limit

$$
\int_{\tau_{k}}^{t} \frac{\partial}{\partial x} f^{0,0}\left(s, \tau_{k}\right) \delta x_{\rho k}(s) d s
$$

where we let $\lim _{\varepsilon \rightarrow 0} z_{k}^{\varepsilon}(\cdot)=\delta x_{\rho k}(\cdot)$. From (3.9) it follows that

$$
\delta x_{\rho k}(t)=\int_{\tau_{k}}^{t}\left\{\frac{\partial}{\partial x} f^{0,0}\left(s, \tau_{k}\right) \delta x_{\rho k}(s)+\Delta f_{k}(s)\right\} d s .
$$

Then

$$
\frac{d}{d t} \delta x_{k}(t)=\frac{\partial}{\partial x} f^{0,0}\left(t, \tau_{k}\right) \delta x_{\rho k}(t)+\Delta f_{k}(t), \quad t \in\left[\tau_{k}, \tau_{k+1}\right) .
$$

Similarly, we consider the discrete event state at $\tau_{k+1}$,

$$
q_{\rho k}^{\iota}\left(\tau_{k+1}\right)=v\left(x_{\rho k}^{\iota}\left(\tau_{k+1}^{-}\right), q_{\rho k}^{0}\left(\tau_{k}\right), u_{\rho k}^{0}\left(\tau_{k+1}^{-}\right)\right), \quad \iota=0, \varepsilon .
$$

We define

$$
\Delta q_{k}(\cdot)=q_{\rho k}^{\varepsilon}(\cdot)-q_{\rho k}^{0}(\cdot) \quad \text { and } \quad \delta q_{\rho k}\left(\tau_{k+1}\right)=\lim _{\varepsilon \rightarrow 0} \frac{1}{\varepsilon} \Delta q_{k}\left(\tau_{k+1}\right),
$$

which denotes the perturbation of the discrete event at $\tau_{k+1}$. We obtain that

$$
\delta q_{\rho k}\left(\tau_{k+1}\right)=\frac{\partial}{\partial x} v\left(x_{\rho k}^{0}\left(\tau_{k+1}\right), q_{\rho k}^{0}\left(\tau_{k}\right), u_{\rho k}^{0}\left(\tau_{k+1}\right)\right) \delta x_{\rho k}\left(\tau_{k+1}\right) .
$$

Over the following $\left[\tau_{h}, \tau_{h+1}\right)(h=k+1, k+2, \ldots, l)$, the variational equation of the HDS's continuous subsystem is written as

$$
\frac{d}{d t} \delta x_{\rho k}(t)=\nabla f^{0,0}\left(t, \tau_{h}\right) \cdot\left(\delta x_{\rho k}(t), \delta q_{\rho k}\left(\tau_{h}\right)\right)
$$


By (3.11) and (3.12) we know that

$$
\begin{aligned}
\delta q_{\rho k}\left(\tau_{h}\right)=\sum_{i=k+1}^{h-1}\left[\prod_{j=0}^{h-i-1} \frac{\partial}{\partial q} v\left(x_{\rho k}^{0}\left(\tau_{h-j}\right), q_{\rho k}^{0}\left(\tau_{h-j-1}\right), u_{\rho k}^{0}\left(\tau_{h-j}\right)\right)\right] \\
\quad \times \frac{\partial}{\partial x} v\left(x_{\rho k}^{0}\left(\tau_{i}\right), q_{\rho k}^{0}\left(\tau_{i-1}\right), u_{\rho k}^{0}\left(\tau_{i}\right)\right) \delta x_{\rho k}\left(\tau_{i}\right) \\
+\frac{\partial}{\partial x} v\left(x_{\rho k}^{0}\left(\tau_{h}\right), q_{\rho k}^{0}\left(\tau_{h-1}\right), u_{\rho k}^{0}\left(\tau_{h}\right)\right) \delta x_{\rho k}\left(\tau_{h}\right),
\end{aligned}
$$

where $h=k+2, k+3, \ldots, l+1$.

We study the $k$ th principal diagonal entry of $J_{\rho}^{2}\left(\boldsymbol{u}_{\rho}^{\varepsilon}(\cdot)\right)-J_{\rho}^{2}\left(\boldsymbol{u}_{\rho}(\cdot)\right)$. It follows that

$$
\begin{aligned}
& J_{k \rho}^{2}\left(u_{\rho k}^{\varepsilon}(\cdot)\right)-J_{k \rho}^{2}\left(u_{\rho k}^{0}(\cdot)\right) \\
& \quad=\left\|G\left(x_{\rho k}^{\varepsilon}\left(t^{f}\right), q_{\rho k}^{\varepsilon}\left(\tau_{l}\right)\right)\right\|^{2}-\left\|G\left(x_{\rho k}^{0}\left(t^{f}\right), q_{\rho k}^{0}\left(\tau_{l}\right)\right)\right\|^{2} \\
& \quad+\left[J_{k}\left(u_{\rho k}^{\varepsilon}(\cdot)\right)-J_{k}(\bar{u}(\cdot))+\sqrt{\rho}\right]^{2}-\left[J_{k}\left(u_{\rho k}^{0}(\cdot)\right)-J_{k}(\bar{u}(\cdot))+\sqrt{\rho}\right]^{2} .
\end{aligned}
$$

For the first two items of the right-hand side of (3.14), we note that

$$
\begin{aligned}
& \left\|G\left(x_{\rho k}^{\varepsilon}\left(t^{f}\right), q_{\rho k}^{\varepsilon}\left(\tau_{l}\right)\right)\right\|^{2}-\left\|G\left(x_{\rho k}^{0}\left(t^{f}\right), q_{\rho k}^{0}\left(\tau_{l}\right)\right)\right\|^{2} \\
& \quad=2\left\langle G\left(x_{\rho k}^{0}\left(t^{f}\right), q_{\rho k}^{0}\left(\tau_{l}\right)\right), \nabla G\left(x_{\rho k}^{0}\left(t^{f}\right), q_{\rho k}^{0}\left(\tau_{l}\right)\right) \cdot\left(\Delta x_{k}\left(t^{f}\right), \Delta q_{k}\left(\tau_{l}\right)\right)+o(\varepsilon)\right\rangle .
\end{aligned}
$$

For the final two terms on the right-hand side of (3.14),

$$
\begin{aligned}
& {\left[J_{k}\left(u_{\rho k}^{\varepsilon}(\cdot)\right)-J_{k}(\bar{u}(\cdot))+\sqrt{\rho}\right]^{2}-\left[J_{k}\left(u_{\rho k}^{0}(\cdot)\right)-J_{k}(\bar{u}(\cdot))+\sqrt{\rho}\right]^{2}} \\
& \quad=2\left[J_{k}\left(u_{\rho k}^{0}(\cdot)\right)-J_{k}(\bar{u}(\cdot))+\sqrt{\rho}\right]\left[J_{k}\left(u_{\rho k}^{\varepsilon}(\cdot)\right)-J_{k}\left(u_{\rho k}^{0}(\cdot)\right)\right]+o(\varepsilon) .
\end{aligned}
$$

According to (3.8), then

$$
\begin{aligned}
2 \varepsilon & \left\langle G\left(x_{\rho k}^{0}\left(t^{f}\right), q_{\rho k}^{0}\left(\tau_{l}\right)\right), \frac{1}{\varepsilon} \nabla G\left(x_{\rho k}^{0}\left(t^{f}\right), q_{\rho k}^{0}\left(\tau_{l}\right)\right) \cdot\left(\Delta x_{k}\left(t^{f}\right), \Delta q_{k}\left(\tau_{l}\right)\right)\right\rangle \\
& +2 \varepsilon\left[J_{k}\left(u_{\rho k}^{0}(\cdot)\right)-J_{k}(\bar{u}(\cdot))+\sqrt{\rho}\right] \frac{1}{\varepsilon}\left[J_{k}\left(u_{\rho k}^{\varepsilon}(\cdot)\right)-J_{k}\left(u_{\rho k}^{0}(\cdot)\right)\right] \\
& +o(\varepsilon)+\varepsilon \rho\left(\tau_{k+1}-\tau_{k}\right) \geq 0 .
\end{aligned}
$$

\section{Defining}

$$
\begin{gathered}
L^{0,0}\left(\cdot, \tau_{k}\right)=L\left(\cdot, x_{\rho k}^{0}(\cdot), q_{\rho k}^{0}\left(\tau_{k}\right), u_{\rho k}^{0}(\cdot)\right), \\
\Delta L_{k}(\cdot)=\left[L\left(\cdot, x_{\rho k}^{0}(\cdot), q_{\rho k}^{0}\left(\tau_{k}\right), u_{k}^{0}(\cdot)\right)-L^{0,0}\left(\cdot, \tau_{k}\right)\right],
\end{gathered}
$$


then by (2.4),

$$
\begin{aligned}
\lim _{\varepsilon \rightarrow 0} \frac{1}{\varepsilon}[ & \left.J_{k}\left(u_{\rho k}^{\varepsilon}(\cdot)\right)-J_{k}\left(u_{\rho k}^{0}(\cdot)\right)\right] \\
= & \int_{\tau_{k}}^{\tau_{k+1}}\left\{\frac{\partial}{\partial x} L^{0,0}\left(s, \tau_{k}\right) \delta x_{\rho k}(s)+\Delta L_{k}(s)\right\} d s \\
& +\sum_{i=k+1}^{l}\left\{\int_{\tau_{i}}^{\tau_{i+1}} \nabla L^{0,0}\left(s, \tau_{i}\right) \cdot\left(\delta x_{\rho k}(s), \delta q_{\rho k}\left(\tau_{i}\right)\right) d s\right. \\
& \left.+\frac{d}{d q} L_{a}\left(q_{\rho k}^{0}\left(\tau_{i}\right)\right) \delta q_{\rho k}\left(\tau_{i}\right)\right\} \\
& +\nabla g\left(x_{\rho k}^{0}\left(t^{f}\right), q_{\rho k}^{0}\left(\tau_{l}\right)\right) \cdot\left(\delta x_{\rho k}\left(t^{f}\right), \delta q_{\rho k}\left(\tau_{l}\right)\right) .
\end{aligned}
$$

Define

$$
\psi_{\rho k}^{\varepsilon}=\frac{2 G\left(x_{\rho k}^{0}\left(t^{f}\right), q_{\rho k}^{0}\left(\tau_{l}\right)\right)}{J_{k \rho}\left(u_{\rho k}^{\varepsilon}(\cdot)\right)+J_{k \rho}\left(u_{\rho k}^{0}(\cdot)\right)} \quad \text { and } \quad h_{\rho k}^{\varepsilon}=\frac{2\left(J_{k}\left(u_{\rho k}^{0}(\cdot)\right)-J_{k}(\bar{u}(\cdot))+\sqrt{\rho}\right)}{J_{k \rho}\left(u_{\rho k}^{\varepsilon}(\cdot)\right)+J_{k \rho}\left(u_{\rho k}^{0}(\cdot)\right)} \text {. }
$$

Then $\lim _{\varepsilon \rightarrow 0}\left(\left\|\psi_{\rho k}^{\varepsilon}\right\|^{2}+h_{\rho k}^{\varepsilon 2}\right)=1$. When $\varepsilon \rightarrow 0$, we have a subsequence of $\left(\psi_{\rho k}^{\varepsilon}, h_{\rho k}^{\varepsilon}\right)$ converging to $\left(\psi_{\rho k}, h_{\rho k}\right)$, which satisfies $\left\|\psi_{\rho k}\right\|^{2}+h_{\rho k}^{2}=1$. By (3.17) and (3.18), taking $\varepsilon \rightarrow 0$ allows (3.16) to be rewritten as

$$
\begin{aligned}
& \left\langle\psi_{\rho k}, \nabla G\left(x_{\rho k}^{0}\left(t^{f}\right), q_{\rho k}^{0}\left(\tau_{l}\right)\right) \cdot\left(\delta x_{\rho k}\left(t^{f}\right), \delta q_{\rho k}\left(\tau_{l}\right)\right)\right\rangle \\
& +h_{\rho k}\left[\int_{\tau_{k}}^{\tau_{k+1}}\left\{\frac{\partial}{\partial x} L^{0,0}\left(s, \tau_{k}\right) \delta x_{\rho k}(s)+\Delta L_{k}(s)\right\} d s\right. \\
& +\sum_{i=k+1}^{l}\left\{\int_{\tau_{i}}^{\tau_{i+1}}\left[\nabla L^{0,0}\left(s, \tau_{i}\right) \cdot\left(\delta x_{\rho k}(s), \delta q_{\rho k}\left(\tau_{i}\right)\right)\right] d s\right. \\
& \left.+\frac{\partial}{\partial q} L_{a}\left(q_{\rho k}^{0}\left(\tau_{i}\right)\right) \delta q_{\rho k}\left(\tau_{i}\right)\right\} \\
& \left.+\nabla g\left(x_{\rho k}^{0}\left(t^{f}\right), q_{\rho k}^{0}\left(\tau_{l}\right)\right) \cdot\left(\delta x_{\rho k}\left(t^{f}\right), \delta q_{\rho k}\left(\tau_{l}\right)\right)\right] \\
& +\frac{\rho\left(\tau_{k+1}-\tau_{k}\right)}{4 J_{k \rho}^{2}\left(u_{\rho k}^{0}(\cdot)\right)} \geq 0 .
\end{aligned}
$$

We define the costate equation of the continuous subsystems as follows. Whenever $t \in\left[\tau_{k}, \tau_{k+1}\right)$, the costate equation of (3.10) is

$$
-\frac{d}{d t} y_{\rho k}(t)=\frac{\partial}{\partial x}\left[f^{0,0}\left(t, \tau_{k}\right) y_{\rho k}(t)+h_{\rho k} L^{0,0}\left(t, \tau_{k}\right)\right] .
$$


Based on the conjugate relation between (3.10) and (3.20),

$$
\begin{aligned}
& \left\langle y_{\rho k}\left(\tau_{k+1}\right), \delta x_{\rho k}\left(\tau_{k+1}\right)\right\rangle-\left\langle y_{\rho k}\left(\tau_{k}\right), \delta x_{\rho k}\left(\tau_{k}\right)\right\rangle \\
& \quad=\int_{\tau_{k}}^{\tau_{k+1}}\left\{-h_{\rho k} \frac{\partial}{\partial x} L^{0,0}\left(s, \tau_{k}\right) \delta x_{\rho k}(s)+\left\langle y_{\rho k}(s), \Delta f_{k}(s)\right\rangle\right\} d s .
\end{aligned}
$$

Whenever $t \in\left[\tau_{h}, \tau_{h+1}\right)$ for $h=k+1, k+2, \ldots, l-1$, or $t \in\left[\tau_{l}, t^{f}\right]$ for $h=l$, the costate equation of (3.12) is

$$
-\frac{d}{d t} y_{\rho k}(t)=\frac{\partial^{T}}{\partial x} f^{0,0}\left(t, \tau_{h}\right) y_{\rho k}(t)+h_{\rho k} \frac{\partial}{\partial x} L^{0,0}\left(t, \tau_{h}\right) .
$$

Then there exists a conjugated relationship between (3.12) and (3.21) with

$$
\begin{aligned}
& \left\langle y_{\rho k}\left(\tau_{h+1}\right), \delta x_{\rho k}\left(\tau_{h+1}\right)\right\rangle-\left\langle y_{\rho k}\left(\tau_{h}\right), \delta x_{\rho k}\left(\tau_{h}\right)\right\rangle \\
& =\int_{\tau_{h}}^{\tau_{h+1}}\left\{-h_{\rho k} \frac{\partial}{\partial x} L^{0,0}\left(s, \tau_{h}\right) \delta x_{\rho k}(s)\right. \\
& \left.\quad+\left\langle\frac{\partial^{T}}{\partial x} f^{0,0}\left(t, \tau_{h}\right) y_{\rho k}(s), \delta q_{\rho k}\left(\tau_{h}\right)\right\rangle\right\} d s .
\end{aligned}
$$

And at the terminal time $t^{f}$, we get

$$
y_{\rho k}\left(t^{f}\right)=\frac{\partial^{T}}{\partial x} G\left(x_{\rho k}^{0}\left(t^{f}\right), q_{\rho k}^{0}\left(\tau_{l}\right)\right) \psi_{\rho k}+h_{\rho k} \frac{\partial}{\partial x} g\left(x_{\rho k}^{0}\left(t^{f}\right), q_{\rho k}^{0}\left(\tau_{l}\right)\right) .
$$

Combining (3.21) with (3.23), we get

$$
\begin{aligned}
\left\langle y_{\rho k}\left(t^{f}\right), \delta x_{\rho k}\left(t^{f}\right)\right\rangle & \\
= & \left\langle y_{\rho k}\left(t^{f}\right), \delta x_{\rho k}\left(t^{f}\right)\right\rangle-\left\langle y_{\rho k}\left(\tau_{l}\right), \delta x_{\rho k}\left(\tau_{l}\right)\right\rangle \\
& +\sum_{h=k}^{l-1}\left\{\left\langle y_{\rho k}\left(\tau_{h+1}\right), \delta x_{\rho k}\left(\tau_{h+1}\right)\right\rangle-\left\langle y_{\rho k}\left(\tau_{h}\right), \delta x_{\rho k}\left(\tau_{h}\right)\right\rangle\right\} \\
= & \sum_{h=k+1}^{l} \int_{\tau_{h}}^{\tau_{h+1}}\left\{-h_{\rho k} \frac{\partial}{\partial x} L^{0,0}\left(s, \tau_{h}\right) \delta x_{\rho k}(s)\right. \\
& \left.+\left\langle\frac{\partial^{T}}{\partial q} f^{0,0}\left(t, \tau_{h}\right) y_{\rho k}(s), \delta q_{\rho k}\left(\tau_{h}\right)\right\rangle\right\} d s \\
& +\int_{\tau_{k}}^{\tau_{k+1}}\left\{-h_{\rho k} \frac{\partial}{\partial x} L^{0,0}\left(s, \tau_{k}\right) \delta x_{\rho k}(s)+\left\langle y_{\rho k}(s), \Delta f_{k}(s)\right\rangle\right\} d s .
\end{aligned}
$$

We assume that $u \in U$ is fixed, and that $t$ is a Lebesgue point of the functions

$$
\psi_{k} L^{0,0}\left(t, \tau_{k}\right)+\left\langle y_{\rho k}(t), f^{0,0}\left(t, \tau_{k}\right)\right\rangle
$$

and

$$
\psi_{k} L\left(t, x_{\rho k}^{0}(t), q_{\rho k}^{0}\left(\tau_{k}\right), u\right)+\left\langle y_{\rho k}(t), f\left(t, x_{\rho k}^{0}(t), q_{\rho k}^{0}\left(\tau_{k}\right), u\right)\right\rangle
$$


Then for $\varepsilon>0$, we take $u_{k}(\cdot) \in U_{\mathrm{ad}}$, defined by

$$
u_{k}(s)= \begin{cases}u, & |s-t| \leq \varepsilon \\ u_{\rho k}(s), & \text { otherwise }\end{cases}
$$

Because $f$ has bounded partial derivatives with respect to $x$ on $\left[\tau_{k}, \tau_{k+1}\right)$, we choose to let the upper bound be $C$ for all $k$. Then $f$ satisfies Lipschitz's condition with respect to $u$. Let $D$ be the Lipschitz constant. Then for $t \in\left[\tau_{k}, \tau_{k+1}\right)$,

$$
\begin{aligned}
\left\|\delta x_{\rho k}(t)\right\| & \leq \int_{\tau_{k}}^{t}\left\|\frac{\partial}{\partial x} f^{0,0}\left(s, \tau_{k}\right)\right\|\left\|\delta x_{\rho k}(s)\right\| d s+\int_{\tau_{k}}^{\tau_{k+1}}\left\|\Delta f_{k}(s)\right\| d s \\
& \leq \int_{\tau_{k}}^{t} C\left\|\delta x_{\rho k}(s)\right\| d s+\int_{t-\varepsilon}^{t+\varepsilon} D\left\|u-u_{\rho k}(s)\right\| d s .
\end{aligned}
$$

As the input control $u_{\rho k}(\cdot) \in U_{\text {ad }}$ is bounded, by Gronwall's inequality [36],

$$
\sup _{t \in\left[\tau_{k}, \tau_{k+1}\right)}\left\{\left\|\delta x_{\rho k}(t)\right\|\right\} \leq C^{\prime} \varepsilon,
$$

where

$$
C^{\prime}=2 D e^{C\left(\tau_{k+1}-\tau_{k}\right)} \max \left\{\left\|u-u_{\rho k}(s)\right\|: u_{\rho k}(\cdot) \in U_{\mathrm{ad}},|s-t| \leq \varepsilon\right\} .
$$

Hence $\lim _{\mathcal{E} \rightarrow 0}\left\|\delta x_{\rho k}\left(\tau_{k+1}\right)\right\|=0$. By (3.11), since $v$ has bounded partial derivatives with respect to $x, \lim _{\varepsilon \rightarrow 0}\left\|\delta q_{\rho k}\left(\tau_{k+1}\right)\right\|=0$. Using (3.12) and (3.13), we discuss all the states of the CVDS and the DEDS at all the following $\tau_{k}$. Finally, we obtain $\lim _{\varepsilon \rightarrow 0}\left\|\delta x_{k}\left(\tau_{h}\right)\right\|=0, \lim _{\varepsilon \rightarrow 0}\left\|\delta q\left(\tau_{h}\right)\right\|=0$, where $h=k+2, k+3, \ldots, l$. Employing (3.19), (3.24) and (3.25), we let $\varepsilon \rightarrow 0$ and derive

$$
\int_{\tau_{k}}^{\tau_{k+1}}\left\{h_{\rho k} \Delta L_{k}(s)+\left\langle y_{\rho k}(s), \Delta f_{k}(s)\right\rangle\right\} d s \geq-\frac{\rho\left(\tau_{k+1}-\tau_{k}\right)}{4 J_{k \rho}^{2}\left(u_{\rho k}(\cdot)\right)} .
$$

Similarly, when $\rho \rightarrow 0$, $\left(\psi_{\rho k}, h_{\rho k}\right)$ have a subsequence converging to $\left(\psi_{k}, h_{k}\right)$, which still satisfies $\left\|\psi_{k}\right\|^{2}+h_{k}^{2}=1$. By (3.4), we get that $\lim _{\rho \rightarrow 0} u_{\rho k}(\cdot)=\bar{u}(\cdot)$, and then $\lim _{\rho \rightarrow 0} \delta x_{\rho k}(\cdot)=\delta x_{k}(\cdot)$. Introducing the costate equations as

$$
\begin{gathered}
-\frac{d}{d t} y_{k}(t)=\frac{\partial^{T}}{\partial x} f\left(t, x_{k}(t), q_{k}\left(\tau_{h}\right), u_{k}(t)\right) y_{k}(t)+h_{k} \frac{\partial}{\partial x} L\left(t, x_{k}(t), q_{k}\left(\tau_{h}\right), u_{k}(t)\right), \\
t \in\left[\tau_{h}, \tau_{h+1}\right) \text { for } h=k, k+1, \ldots, l-1 \quad \text { or } t \in\left[\tau_{l}, t^{f}\right] \text { for } h=l,
\end{gathered}
$$

at the terminal instant $t^{f}$, we obtain

$$
y_{k}\left(t^{f}\right)=\frac{\partial^{T}}{\partial x} G\left(x_{k}\left(t^{f}\right), q_{k}\left(\tau_{l}\right)\right) \psi_{k}+h_{k} \frac{\partial}{\partial x} g\left(x_{k}\left(t^{f}\right), q_{k}\left(\tau_{l}\right)\right) .
$$


Then we obtain easily $\lim _{\rho \rightarrow 0} y_{\rho k}(\cdot)=y_{k}(\cdot)$. According to (3.27), whenever $\rho \rightarrow 0$,

$$
\begin{aligned}
& \int_{\tau_{k}}^{\tau_{k+1}}\left\{h_{k}\left[L\left(s, x_{k}(s), q_{k}\left(\tau_{k}\right), u_{k}(s)\right)-L\left(s, x_{k}(s), q_{k}\left(\tau_{k}\right), \bar{u}(s)\right)\right]\right. \\
& \left.\quad+\left\langle y_{k}(s), f\left(s, x_{k}(s), q_{k}\left(\tau_{k}\right), u_{k}(s)\right)-f\left(s, x_{k}(s), q_{k}\left(\tau_{k}\right), \bar{u}(s)\right)\right\rangle\right\} d s \geq 0 .
\end{aligned}
$$

By (3.26), we get

$$
\begin{aligned}
& \int_{t-\varepsilon}^{t+\varepsilon}\left\{h_{k} L\left(s, x_{k}(s), q_{k}\left(\tau_{k}\right), u\right)+\left\langle y_{k}(s), f\left(s, x_{k}(s), q_{k}\left(\tau_{k}\right), u\right)\right\rangle\right\} d s \\
& \geq \int_{t-\varepsilon}^{t+\varepsilon}\left\{h_{k} L\left(s, x_{k}(s), q_{k}\left(\tau_{k}\right), \bar{u}(s)\right)\right. \\
& \left.\quad+\left\langle y_{k}(s), f\left(s, x_{k}(s), q_{k}\left(\tau_{k}\right), \bar{u}(s)\right)\right\rangle\right\} d s .
\end{aligned}
$$

On dividing both sides of (3.28) by $2 \varepsilon$ and letting $\varepsilon \rightarrow 0$, it follows that

$$
\begin{aligned}
& \quad h_{k} L\left(t, x_{k}(t), q_{k}\left(\tau_{k}\right), u\right)+\left\langle y_{k}(t), f\left(t, x_{k}(t), q_{k}\left(\tau_{k}\right), u\right)\right\rangle \\
& \quad \geq h_{k} L\left(t, x_{k}(t), q_{k}\left(\tau_{k}\right), \bar{u}(t)\right)+\left\langle y_{k}(t), f\left(t, x_{k}(t), q_{k}\left(\tau_{k}\right), \bar{u}(t)\right)\right\rangle .
\end{aligned}
$$

Because the set of all Lebesgue points can be fully measured over $\left[\tau_{k}, \tau_{k+1}\right)$ or over $\left[\tau_{l}, t^{f}\right]$, the aforementioned inequality is true almost everywhere on $\left[\tau_{k}, \tau_{k+1}\right)$ or on $\left[\tau_{l}, t^{f}\right]$. Based on the generality of $k$ and the identity of the HDS's trajectory corresponding to the optimal control $\overline{\boldsymbol{u}}(\cdot)$, we obtain that the minimum condition (3.2) holds a.e. $t \in\left[\tau_{k}, \tau_{k+1}\right)$ or $t \in\left[\tau_{l}, t^{f}\right]$. This concludes the proof.

In [10], the optimal control problem of HDSs with restricted terminal states was studied and the minimum principle established. That is, for a class of HDSs, if there is an optimal control input, then we propose a minimum principle over a certain continuous time interval. However, the result is local instead of globally evolved over time. In the theorem proposed in this section, we have generalized the previous results and have stated a minimum principle of global HDSs. In the proof, we have constituted the cost functional as a diagonal matrix, where each diagonal entry corresponds to the cost function on the relevant continuous time interval. Here we have concentrated on the effects of switches in the system. Specially, if the dimension of the matrix is one, in other words, the cost functional is scalar, the above principle reduces to the classical formulation of Pontryagin's maximum principle for pure continuous systems [36]. Then the cost functional is changed to

$$
J(u(\cdot))=\int_{0}^{t^{f}} L(s, x(s, u(s)), u(s)) d s+g\left(x\left(t^{f}, u(\cdot)\right), q\left(\tau_{l}, u(\cdot)\right)\right) .
$$

The dynamics of the continuous system are defined by

$$
\dot{x}(t)=f(t, x(t), u(t)),
$$

and the restricted condition of the system's terminal state is $G\left(x\left(t^{f}\right)\right)=0$. Then we obtain the classical formulation of the minimum principle for pure continuous systems as follows. 
COROLlaRY 3.4. Let $\bar{u}(\cdot)$ be a solution to the optimal control problem of system (3.29). Suppose that $x(\cdot)=x(\cdot, \bar{u}(\cdot))$ is the optimal trajectory of the system corresponding to the optimal control $\bar{u}(\cdot)$. Then over the evolving interval $\left[0, t^{f}\right]$ there exist a scalar $h$ and piecewise continuous $y_{k}(\cdot)$ that satisfy the adjoint equation

$$
-\frac{d}{d t} y(t)=\frac{\partial^{T}}{\partial x} f(t, x(t), \bar{u}(t)) y(t)+h \frac{\partial}{\partial x} L(t, x(t), \bar{u}(t)) .
$$

There exists an $n_{1}$-dimensional $\psi$ such that $h^{2}+\|\psi\|^{2}=1$. At the terminal instant $t^{f}$,

$$
y\left(t^{f}\right)=\frac{\partial^{T}}{\partial x} G\left(x\left(t^{f}\right)\right) \psi+h \frac{\partial}{\partial x} g\left(x\left(t^{f}\right)\right) .
$$

At the same time, the minimum condition

$$
\begin{aligned}
& h L(t, x(t), \bar{u}(t))+\langle y(t), f(t, x(t), \bar{u}(t))\rangle \\
& \quad=\min _{u \in U}\{h L(t, x(t), u)+\langle y(t), f(t, x(t), u)\rangle\}, \quad \text { a.e. } t \in\left[0, t^{f}\right],
\end{aligned}
$$

is satisfied on the same interval.

\section{Application towards linear switched systems and pure discrete-time systems}

We consider a class of switched systems composed of limited controlled subsystems. The system is described as

$$
\begin{cases}\dot{x}(t)=A_{q\left(\tau_{k}\right)}(t) x(t)+B_{q\left(\tau_{k}\right)}(t) u(t), & t \in\left[\tau_{k}, \tau_{k+1}\right) \text { or } t \in\left[\tau_{l}, t^{f}\right], \\ q\left(\tau_{k+1}\right)=v\left(x\left(\tau_{k+1}\right), q\left(\tau_{k}\right), u\left(\tau_{k+1}\right)\right), & k=0,1, \ldots, l,\end{cases}
$$

where $A_{\left.q_{(} \tau_{k}\right)}(t)$ and $B_{\left.q_{(\tau}\right)}(t)$ are matrices of appropriate dimensions and we suppose that $\tau_{l+1}=t^{f}$. The restricted condition of the system's terminal state is

$$
x^{T}\left(t^{f}\right) W_{f} x\left(t^{f}\right)+q^{T}\left(\tau_{l}\right) M_{f} q\left(\tau_{l}\right)=c,
$$

where $W_{f}$ and $M_{f}$ are positive-definite symmetric matrices of appropriate dimensions and $c$ is a positive real number. The $k$ th diagonal entry of the evolving cost functional of (4.1) is defined by

$$
\begin{array}{r}
J_{k}\left(u_{k}(\cdot)\right)=\sum_{i=k}^{l} \int_{\tau_{i}}^{\tau_{i+1}} \frac{1}{2}\left[x_{k}^{T}(s) Q_{k} x_{k}(s)+u_{k}^{T}(s) V_{k} u_{k}(s)\right] d s \\
+\sum_{i=k+1}^{l} L_{a}\left(q\left(\tau_{i}, u_{k}(\cdot)\right)\right)+x_{k}^{T}\left(t^{f}\right) R x_{k}\left(t^{f}\right),
\end{array}
$$

where $Q_{k}, V_{k}$ and $R$ are positive-definite symmetric matrices. Then the minimum principle for (4.1) can be stated as follows. 
THEOREM 4.1. Let $\bar{u}(\cdot)$ be a solution of the optimal control problem for (4.1). We suppose that $x(\cdot)=x(\cdot, \bar{u}(\cdot))$ is the optimal continuous trajectory and $q(\cdot)=$ $q(\cdot, \bar{u}(\cdot))$ is the optimal discrete event state corresponding to $\bar{u}(\cdot)$. Then over $\left[\tau_{0}, t^{f}\right]$, there exist a scalar $h_{k}$ and piecewise continuous $y_{k}(\cdot)$ that satisfy the adjoint equation

$$
-\frac{d}{d t} y_{k}(t)=A_{q\left(\tau_{k}\right)}(t) y_{k}(t)+h_{k} Q_{k} x_{k}(t)
$$

over all $\left[\tau_{k}, \tau_{k+1}\right)$ for $k=0,1, \ldots, l-1$, or over $\left[\tau_{l}, t^{f}\right]$ for $k=l$. There exist scalars $\psi_{k}(k=0,1,2, \ldots, l)$ such that $h_{k}^{2}+\psi_{k}^{2}=1$. At $t^{f}, y_{k}(\cdot)$ satisfies

$$
y_{k}\left(t^{f}\right)=W_{f} x\left(t^{f}\right) \psi_{k}+h_{k} R x\left(t^{f}\right) .
$$

At the same time, the minimum condition

$$
\begin{array}{r}
H\left(t, x(t), q\left(\tau_{k}\right), y_{k}(t), \bar{u}(t)\right)=\min _{u \in U} H\left(t, x(t), q\left(\tau_{k}\right), y_{k}(t), u\right), \\
\text { a.e. } t \in\left[\tau_{k}, \tau_{k+1}\right) \text { or }\left[\tau_{l}, t^{f}\right],
\end{array}
$$

holds, where

$$
\begin{aligned}
H\left(t, x(t), q\left(\tau_{k}\right), y_{k}(t), u(t)\right)= & \frac{1}{2} h_{k}\left[x^{T}(t) Q_{k} x(t)+u^{T}(t) V_{k} u(t)\right] \\
& +y_{k}^{T}(t)\left[A_{q\left(\tau_{k}\right)}(t) x(t)+B_{q\left(\tau_{k}\right)}(t) u(t)\right] .
\end{aligned}
$$

REMARK 3. In the case of $u \in U$, the minimum condition (4.2) and (4.3) stated in Theorem 4.1 can be expressed as

$$
\min _{u \in U}\left\{\frac{1}{2} h_{k} u^{T} V_{k} u+y_{k}^{T}(t) B_{q\left(\tau_{k}\right)}(t) u\right\}=\frac{1}{2} h_{k} \bar{u}^{T}(t) V_{k} \bar{u}(t)+y_{k}^{T}(t) B_{q\left(\tau_{k}\right)}(t) \bar{u}(t) .
$$

From the minimum condition of (4.4), we get the optimal control input over the $k$ th interval by differential calculation. Let

$$
\tilde{H}\left(y_{k}(t), u(t)\right)=\frac{1}{2} h_{k} u^{T}(t) V_{k} u(t)+y_{k}^{T}(t) B_{q\left(\tau_{k}\right)}(t) u(t) .
$$

Then differentiate $\tilde{H}$ at $u(t)=\bar{u}(t)$,

$$
0=\left.\frac{d}{d u} \tilde{H}\right|_{u=\bar{u}(t)}=h_{k} V_{k} \bar{u}(t)+y_{k}^{T}(t) B_{q\left(\tau_{k}\right)}(t),
$$

and we obtain the optimal control input as

$$
\bar{u}(t)=-\frac{1}{h_{k}} V_{k}^{-1} y_{k}^{T}(t) B_{q\left(\tau_{k}\right)}(t), \quad t \in\left[\tau_{k}, \tau_{k+1}\right) .
$$

REMARK 4. If the HDS (4.1) reduces to a pure linear time-variant system with no switching, described by

$$
\dot{x}(t)=A(t) x(t)+B(t) u(t), \quad t \in\left[0, t^{f}\right),
$$


then we suppose that the evolving cost functional of (4.5) corresponds to its potential energy. That is, the cost functional is defined by its states as

$$
J(u(\cdot))=\int_{0}^{t^{f}} x^{T}(s, u(\cdot)) Q x(s, u(\cdot)) d s+x^{T}\left(t^{f}\right) R x\left(t^{f}\right),
$$

which is the minimum principle of pure continuous linear time-invariant systems. This is similar to Theorem 3.3 so we will omit it here, but we note that the optimal control will be written in a simpler form for the systems and cost functional above. The new Hamiltonian will be denoted by

$$
H(t, x(t), y(t), u(t))=h x^{T}(t) Q x(t)+y^{T}(t)[A(t) x(t)+B(t) u(t)] .
$$

In (4.7) $x(t)$ is the optimal trajectory corresponding to $\bar{u}(t)$, and so the minimum condition becomes

$$
\min _{u \in U}\left\{y^{T}(t) B(t) u\right\}=y^{T}(t) B(t) \bar{u}(t) .
$$

Here we let $\varphi(t)=y^{T}(t) B(t)=\left(\varphi_{1}(t), \varphi_{2}(t), \ldots, \varphi_{m}(t)\right)$. Then by the definition of $U$, there exists

$$
\min _{u \in U} \varphi(t) u=\sum_{i=1}^{m} \min _{|u| \leq 1} \varphi_{i}(t) u_{i}=-\sum_{i=1}^{m}\left|\varphi_{i}(t)\right| .
$$

Then the $i$ th component of the optimal control $\bar{u}(t)$ can be denoted by $\bar{u}_{i}(t)=$ $-\operatorname{sign}\left(\varphi_{i}(t)\right)$, where the function sign denotes that the component of the optimal control will take values in the set $\{-1,1\}$. Then it follows that we can solve the optimal control problem of a pure continuous linear time-invariant system using a "switching control".

Our result can also be used in the case of pure discrete-time systems. Since the class of systems considers their dynamics only at the discrete sampling instants, we state the dynamics of pure discrete-time systems with fixed switches as

$$
q\left(\tau_{k+1}\right)=v\left(\tau_{k+1}, q\left(\tau_{k}\right), u\left(\tau_{k+1}\right)\right), \quad k=0,1,2, \ldots, l-1,
$$

where we let $\tau_{0}=0$. Then the discrete-time systems run over the interval $\left[0, t^{f}\right]$. We suppose that there exists a discrete admissible control sequence $\left.u\left(\tau_{k}\right)\right|_{k=0,1, \ldots, l}$, under which the terminal state of (4.1) yields $G\left(q\left(t^{f}\right)\right)=0$. We define the cost functional

$$
J(u(\cdot))=\sum_{i=0}^{l+1} L\left(\tau_{k}, q\left(\tau_{k}\right), u\left(\tau_{k}\right)\right)+g\left(q\left(t^{f}\right)\right) .
$$

Then by Theorem 3.3, we get the minimum principle for discrete-time systems.

Corollary 4.2. System (4.8) is a discrete-time system. Let $\left.\bar{u}\left(\tau_{k}\right)\right|_{k=0,1, \ldots, l}$ be a solution. We suppose that $q\left(\tau_{k}\right)=q\left(\tau_{k}, \bar{u}\left(\tau_{k-1}\right)\right)$ is the optimal state of system (4.8) 
corresponding to the optimal control $\bar{u}\left(\tau_{k}\right)$. Then over the global evolving interval $\left[0, t^{f}\right]$, we get the discrete adjoint equation

$$
\begin{gathered}
\lambda\left(\tau_{k+1}\right)=\lambda\left(\tau_{k}\right)-\left(\tau_{k+1}-\tau_{k}\right)\left[\frac{\partial}{\partial q} v\left(\tau_{k+1}, q\left(\tau_{k}\right), \bar{u}\left(\tau_{k+1}\right)\right) \lambda\left(\tau_{k}\right)\right. \\
\left.+h \frac{\partial}{\partial q} L\left(\tau_{k+1}, q\left(\tau_{k}\right), \bar{u}\left(\tau_{k+1}\right)\right)\right] .
\end{gathered}
$$

There exists $\psi$ such that $h^{2}+\|\psi\|^{2}=1 . \lambda\left(t^{f}\right)$ satisfies transversal conditions

$$
\lambda\left(t^{f}\right)=\frac{d^{T}}{d q} G\left(q\left(t^{f}\right)\right) \psi+h \frac{d}{d q} v\left(q\left(t^{f}\right)\right) .
$$

At every switching instant, the minimum conditions are satisfied. That is,

$$
H\left(\tau_{k}, q\left(\tau_{k}\right), y\left(\tau_{k+1}\right), \bar{u}\left(\tau_{k}\right)\right)=\min _{u \in U} H\left(\tau_{k}, q\left(\tau_{k}\right), y\left(\tau_{k+1}\right), u\right),
$$

where

$$
\begin{aligned}
& H\left(\tau_{k}, q\left(\tau_{k}\right), \lambda\left(\tau_{k+1}\right), u\left(\tau_{k}\right)\right) \\
& \quad=h L\left(\tau_{k}, q\left(\tau_{k}\right), u\left(\tau_{k}\right)\right)+\left\langle\lambda\left(\tau_{k+1}\right), v\left(\tau_{k}, q\left(\tau_{k}\right), u\left(\tau_{k}\right)\right)\right\rangle .
\end{aligned}
$$

REMARK 5. Through the above applications, we see that the result proposed in this paper covers both pure continuous systems and pure discrete-time systems. The HDS model put forward here is a powerful model, and the theoretical solution of its optimal control problems, the hybrid minimum principle, is a generalization from the optimal control problems of two classical systems.

\section{Conclusions}

In this paper, we have introduced an optimal control problem for a general class of global hybrid dynamical systems with restricted terminal states. We have established the necessary conditions for the aforementioned optimal control problem, the minimum principle of global HDSs, and then proved the theorem. In the proof, Ekeland's variational principle and matrix cost functional structure expression are applied. The results offer a research method to deal with optimal control problems of switched systems.

\section{Acknowledgements}

The authors would like to thank the editor and the reviewers for their constructive comments and valuable suggestions which improved the quality of the paper. This work was supported in part by the China Postdoctoral Science Foundation under grant 20090451314, the Outstanding Young Scientists Incentive Funding of Shandong Province under grant BS2009DX041, the Postdoctoral Creative Foundation of Shandong Province under grant 200703085, and NSERC Canada. 


\section{References}

[1] M. Baotic, F. J. Christophersen and M. Morari, "Constrained optimal control of hybrid systems with a linear performance index", IEEE Trans. Automat. Control 51 (2006) 1903-1919.

[2] A. Bemporad and M. Morari, "Control of systems integrating logic, dynamics, and constraints", Automatica 35 (1999) 407-427.

[3] S. C. Bengea and R. A. DeCarlo, "Optimal control of switching systems", Automatica 41 (2005) $11-27$.

[4] G. Borrelli, M. Baotic, A. Bemporad and M. Morari, "Dynamic programming for constrained optimal control of discrete-time linear hybrid systems", Automatica 41 (2006) 1709-1721.

[5] M. S. Branicky, "Studies in hybrid systems: modeling, analysis and control", Ph.D. Dissertation, Massachusetts Institute of Technology, 1995.

[6] M. S. Branicky, "Lyapunov functions and other analysis tools for switched and hybrid systems", IEEE Trans. Automat. Control 43 (1998) 475-482.

[7] M. S. Branicky, V. S. Borkar and S. K. Mitter, "A unified framework for hybrid control: model and optimal control theory", IEEE Trans. Automat. Control 43 (1998) 31-45.

[8] C. G. Cassandras, D. L. Pepyne and Y. Wardi, "Optimal control of a class of hybrid systems", IEEE Trans. Automat. Control 46 (2001) 398-415.

[9] C. H. Chai and A. R. Teel, "Smooth Lyapunov functions for hybrid systems. Part I: existence is equivalent to robustness", IEEE Trans. Automat. Control 54 (2006) 1264-1277.

[10] R. Gao and Y. Z. Wang, "Study on optimal control of HDS by Ekeland's variational principle", Chin. J. Electron. 15 (2006) 487-491.

[11] R. Gao, L. Wang and Y. Z. Wang, "Study of optimal control problems for hybrid dynamical systems", J. Syst. Eng. Electron. 17 (2006) 147-155.

[12] A. Giua, C. Seatzu and C. Van Der Mee, "Optimal control of switched autonomous linear systems", Proceedings of the 40th IEEE Conference on Decision \& Control 12 (2001) 2472-2477.

[13] K. Gokbayrak and O. Selvi, "Optimal hybrid control of a two-stage manugacturing system", in Proceedings of the 2006 American Control Conference, 2006, 3364-3369.

[14] Z. H. Guan, D. J. Hill and X. M. Shen, "On hybrid impulsive and switching systems and application to nonlinear control", IEEE Trans. Automat. Control 50 (2005) 1058-1062.

[15] S. Hedlund and A. Rantzer, "Optimal control of hybrid systems", Proceedings of the 38th IEEE Conference on Decision \& Control 10 (1999) 3972-3976.

[16] M. Lazar, W. P. M. H. Heemels, S. Weiland and A. Bemporad, "Stabilizing model predictive control of hybrid systems", IEEE Trans. Automat. Control 51 (2006) 1813-1818.

[17] X. Z. Liu and J. H. Shen, "Stability theory of hybrid dynamical systems with time delay", IEEE Trans. Automat. Control 54 (2006) 620-625.

[18] Y. Liu, K. L. Teo, L. S. Jennings and S. Wang, "On a class of optimal control problems with state jumps", J. Optim. Theory Appl. 98 (1998) 65-82.

[19] R. C. Loxton, K. L. Teo and V. Rehbock, "Computational method for a class of switched system optimal control problems", IEEE Trans. Automat. Control 54 (2009) 2455-2460.

[20] R. C. Loxton, K. L. Teo, V. Rehbock and W. K. Ling, "Optimal switching instants for a switchedcapacitor DC/DC power converter", Automatica 45 (2009) 973-980.

[21] M. Margaliot, "Stability analysis of switched systems using variational principles: an introduction", Automatica 42 (2006) 2059-2077.

[22] B. Meng and J. F. Zhang, "Output feedback based admissible control of switched linear singular system”, Acta Automat. Sinica 32 (2006) 179-185.

[23] A. N. Michel and B. Hu, "Towards a stability theory of general hybrid dynamical systems", Automatica 35 (1999) 371-384.

[24] D. L. Pepyne and C. G. Cassandras, "Modeling analysis and optimal control of a class of hybrid systems", Discrete Event Dyn. Syst. 8 (1998) 175-201.

[25] D. L. Pepyne and C. G. Cassandras, "Optimal control of hybrid systems in manufacturing", Proc. IEEE 88 (2000) 1108-1123. 
[26] B. D. Schutter, "Optimal control of a class of linear hybrid systems with saturation", Proceedings of the 38th IEEE Conference on Decision \& Control 10 (1999) 3978-3983.

[27] M. S. Shaikh and P. E. Caines, "On the hybrid optimal control problem: theory and algorithms", IEEE Trans. Automat. Control 52 (2007) 1587-1603.

[28] W. Spinelli, P. Bolzern and P. Colaneri, "A note on optimal control of autonomous switched systmes on a finite time interval", in Proceedings of the 2006 American Control Conference, 2006, 5948-5952.

[29] G. Stikkel, J. Bokaor and Z. Szabo, "Necessary and sufficient condition for the controllability of switching linear hybrid systems", Automatica 40 (2004) 1093-1097.

[30] Y. Sun, A. N. Michel and G. S. Zhai, "Stability of discontinuous retarded functional differential equations with applications", IEEE Trans. Automat. Control 50 (2005) 1090-1105.

[31] S. P. Tan, J. F. Zhang and L. L. Yao, "Optimality analysis of adaptive sampled control of hybrid systems with quadratic index", IEEE Trans. Automat. Control 50 (2005) 1044-1051.

[32] G. F. Trecate, F. A. Cuzzola, D. Mignone and M. Morari, "Analysis of discrete-time piecewise affine and hybrid systems", Automatica 38 (2002) 2139-2146.

[33] X. P. Xu and G. S. Zhai, "Practical stability and stabilization of hybrid and switched systems", IEEE Trans. Automat. Control 50 (2005) 1897-1903.

[34] Z. Y. Yang, "An algebraic approach towards the controllability of controlled switching linear hybrid systems", Automatica 38 (2002) 1221-1228.

[35] H. Ye, A. N. Michel and L. Hou, "Stability theory for hybrid dynamical systems", IEEE Trans. Automat. Control 43 (1998) 461-474.

[36] X. M. Zhang, X. J. Li and Z. H. Chen, The differential equation theory of optimal control systems, 1st edn (Higher Education Press, Beijing, 1989). 